\title{
EXTRINSIC CALIBRATION OF A FISHEYE CAMERA AND INERTIAL MEASUREMENT UNIT
}

\author{
LIANG WANG, HE-HUA JU \\ School of Electronic Information and Control Engineering, Beijing University of Technology, Beijing, 100124, China \\ E-MAIL: \{wangliang,juhehua\}@bjut.edu.cn
}

\begin{abstract}
:
Due to its robust performance and wide potential application, the fusion of visual and inertial sensors for motion estimation has attracted significant attention recently. For this fusion, extrinsic calibration is a prerequisite. But most existing methods need a special configuration in which the camera can observe the inertial sensor. In this paper we present an extrinsic calibration method for visual and inertial sensors in a general configuration in which the visual and inertial sensors can not observe each other. The proposed method introduces a total station as a reference. The total station can be adjusted to be parallel to the ground-plane to which the inertial sensor can provide the rotation. Then the rotation between the fisheye camera and the total station is deduced via a homography. Finally the rotation between the fisheye camera and the inertial measurement unit can be obtained. We implement the proposed method on our lunar rover prototype. Experiment results show the validity of the proposed method.
\end{abstract}

\section{Keywords:}

Extrinsic calibration; Fisheye camera; Inertial measurement unit; Homography

\section{Introduction}

Estimating the motion of a rigid body is a long-standing, difficult problem [1, 2, 3, 4]. Inertial Measurement Unit (IMU), which typically measures the first derivative of orientation and the second derivative of position, can be used to perform this task by dead reckoning. A major disadvantage of inertial sensors is that they typically suffer from accumulated error [1]. Because the IMU is continually adding detected changes to its previously calculated positions, any errors in measurement, however small, are accumulated from point to point. This leads to 'drift', or an ever-increasing difference between where the IMU thinks it is located, and the actual location. Finally error may be accumulated enough large to collapse the position and orientation estimation.

Other systems are used to overcome the deficiencies that IMUs inevitably suffer, such as GPS, gravity sensors, a barometric system, and magnetometers [5, 6, 7]. However, gravity sensors and magnetometers are accurate only when the IMU is static and when the magnetic field is stable respectively. Some satellites are needed for GPS and the barometric system only valid when there is atmosphere. For lunar exploration, those systems do not work due to Moon's especial environment, where there is no atmosphere, the gravity is low and the intensity of magnetic field is small. So IMUs have to resort to other instruments to improve measurements.

Camera can also be used to perform motion estimation by identifying and matching man-made or natural landmarks $[3,4,8,9]$. There are a lot of algorithms can be used to perform motion estimation and yield 3D information. Additionally cameras are light, cheap and power saving. All of these make camera can be used in a wide variety of applications. So a fisheye camera is used in our lunar rover prototype as shown in Figure 1. However camera is susceptible to difficulty in identifying and matching good landmarks in some cases.

Fusing IMU and camera data can overcome the drawbacks of each type of sensor to obtain navigation results better than can be obtained from either type of sensor used alone. In order to effectively use data from IMU and camera, it is important to know the relative orientation from each other, which affects the geometric interpretation of their measurements. There are some works on this problem[10,11], but most of them need the camera and the IMU should be in a special configuration where the camera can observe the IMU or object adhered to the IMU to determine their relative orientation. Actually in real application, this configuration is not easy to obtain. A method for the extrinsic calibration of a camera and IMU, which are in a general configuration in which the visual and inertial sensors can not observe each other, is proposed. This work assumes that the internal parameters of camera and initial alignment parameters of IMU are known or can be determined by other methods in advance. The method employs a planar calibration pattern and a total station. The 
planar pattern in a certain pose can constraint the extrinsic parameters between the camera and the total station by points of the planar pattern viewed simultaneously by the camera and the total station. With the total station serving as a reference, the rigid rotation from the camera coordinate system to the IMU coordinate system can be identified.

The rest of the paper is organized as follows. Some preliminaries are introduced in Section II, and Section III gives the proposed extrinsic calibration method in detail. Finally, the preliminary results showing the success of the presented technique and conclusion are presented in Section IV and Section V.

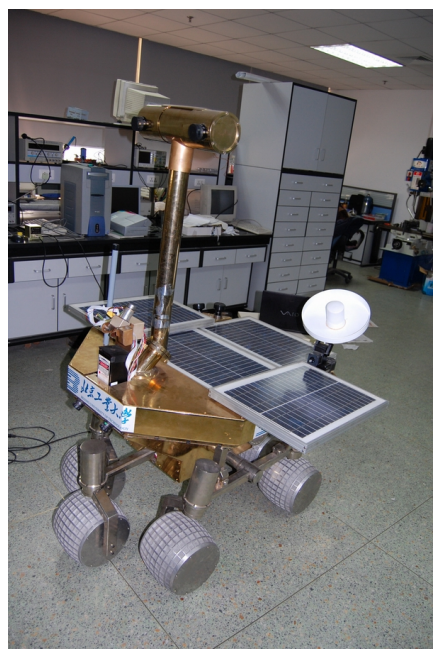

Figure 1. Illustration of the lunar rover prototype.

\section{Preliminaries}

\subsection{IMU}

An IMU works by detecting the current rate of acceleration, as well as changes in rotational attributes, including pitch, roll and yaw. This data is then fed into a computer, which calculates the current speed and position, given a known initial speed and position.

In our system the used IMU is a box containing three accelerometers and three gyroscopes. The accelerometers are placed such that their measuring axes are orthogonal to each other. Three gyroscopes are placed in a similar orthogonal pattern, measuring rotational position in reference to an arbitrarily chosen coordinate system.

\subsection{Fisheye camera}

The view angle of a general camera is about 70 degrees at most, with which only a limited view is covered and result in information losing. The direct way to overcome this deficiency is to employ several cameras, which will make the payload and the complexity of processing method dramatically increasing. In our system fisheye cameras with a view angle of 185 degrees are used, which can overcome the visual information losing problem.
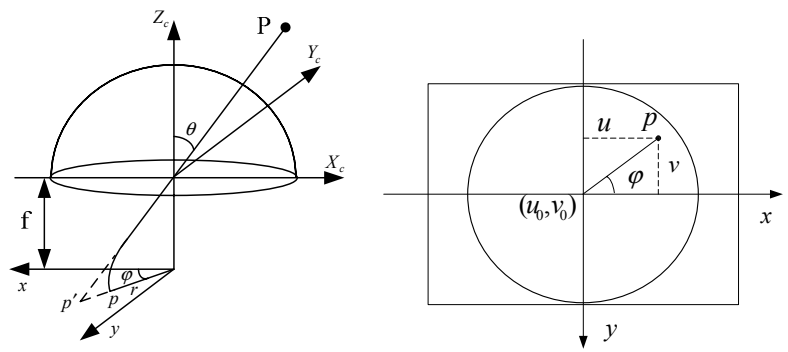

Figure 2. Illustration of fisheye camera.

(a) Projection and (b) Image of fisheye camera. The image of point $P$ is $p$ whereas it would be $p^{\prime}$ by a pinhole camera. $(\theta, \varphi)$ is the incidence angle of $P . r$ is the distance between the image point $p$ and the principal point $\left(u_{0}, v_{0}\right) . f$ is the focal length.

The projection procedure of fisheye camera shown in Figure 2 consists of three steps [9]:

Step 1: Transform the world coordinates $X$ of the space point $P$ into an incoming direction $(\theta, \varphi)$ in camera coordinate system with the relative rotation $R$ and translation $T . \theta$ is the angle between the incoming ray and the optical axis of fisheye camera, $\varphi$ is the angle between the incoming ray and the $\mathrm{x}$-axis of fisheye camera coordinate system.

$$
\left[\begin{array}{lll}
\sin \theta \cos \varphi & \sin \theta \sin \varphi & \cos \theta
\end{array}\right]^{T}=(R X+T) /\|R X+T\|
$$

Step 2: The normalized coordinates $(x, y)$ can be computed from the direction $(\theta, \varphi)$. Here the general form of fisheye lenses [7] is considered

$$
r=k_{1} \theta+k_{2} \theta^{3}+k_{3} \theta^{5} .
$$

Then normalized image coordinates can be expressed as

$$
\left[\begin{array}{ll}
x & y
\end{array}\right]^{\prime}=\left(k_{1} \theta+k_{2} \theta^{3}+k_{3} \theta^{5}\right)\left[\begin{array}{ll}
\cos \varphi & \sin \varphi
\end{array}\right]^{\prime} .
$$

Step 3: Apply affine transformation to the image coordinates. Assuming that the pixel coordinate system is orthogonal, we get its pixel coordinates 


$$
\left[\begin{array}{l}
u \\
v
\end{array}\right]=\left[\begin{array}{cc}
f_{u} & 0 \\
0 & f_{v}
\end{array}\right]\left[\begin{array}{l}
x \\
y
\end{array}\right]+\left[\begin{array}{l}
u_{0} \\
v_{0}
\end{array}\right]
$$

where $f_{u}, f_{v}$ is the focus along horizontal and vertical direction respectively, and $\left(u_{0}, v_{0}\right)$ the principal point.

So the projection model of fisheye camera can be expressed as

$$
\left[\begin{array}{l}
u \\
v
\end{array}\right]=\left[\begin{array}{cc}
f_{u} & 0 \\
0 & f_{v}
\end{array}\right]\left(\left(k_{1} \theta+k_{2} \theta^{3}+k_{3} \theta^{5}\right)\left[\begin{array}{c}
\cos \varphi \\
\sin \varphi
\end{array}\right]+\left[\begin{array}{c}
u_{0} \\
v_{0}
\end{array}\right]\right.
$$

With this model, the direction of an incoming ray $(\theta, \varphi)$ can be determined from its pixel coordinates $(u, v)$ :

$$
\begin{gathered}
{\left[\begin{array}{l}
x \\
y
\end{array}\right]=\left(\left[\begin{array}{cc}
f_{u} & 0 \\
0 & f_{v}
\end{array}\right)^{-1}\left(\left[\begin{array}{l}
u \\
v
\end{array}\right]-\left[\begin{array}{l}
u_{0} \\
v_{0}
\end{array}\right]\right) .\right.} \\
\varphi=a \tan 2(y, x) \\
k_{1} \theta+k_{2} \theta^{3}+k_{3} \theta^{5}=\operatorname{sqrt}\left(x^{2}+y^{2}\right)
\end{gathered}
$$

\section{Solving Extrinsic Calibration}

In our lunar rover, the IMU is built in the inner. The fisheye camera can not observe the IMU. Additionally due to working passively, IMU can not provide any information of the fisheye camera. So there is no direct way to determine the relative orientation between them.

A total station is introduced in our method. The total station can provide the orientation and position of an object in the total station coordinate system in a way similar to fisheye camera. It should be adjusted to be parallel to the ground-plane coordinate system before measurement. The reference coordinate system of IMU is also parallel to the ground-plane coordinate system. So we introduce a total station as a reference to calibrate the relative orientation between the fisheye camera and the IMU.

A 2D planar pattern, say a checker board is placed in front of our system, which is visible to both the camera and the total station. Figure 3 provides a general setup of this calibration method. In the following, we use a calibration plane to refer to the plane surface defined by the checker board for simplicity. And we use control point to refer to the feature point used for calibration on the checker board.

Without loss of generality, we assume that the calibration plane is the plane $\mathrm{Z}=0$ in the world coordinate system. With control point's image coordinates $\left[\begin{array}{ll}u & v\end{array}\right]$ ', we can determine the coordinates $\left[\begin{array}{lll}\sin \theta \cos \varphi & \sin \theta \sin \varphi & \cos \theta\end{array}\right]$ ' of its back-projection in the fisheye camera coordinate system via equation $(6,7,8)$. And these back-projections of control points' images distribute on a unit sphere. Because the transformation from the calibration plane to the unit sphere is a projection, there is a homography $H_{p 2 f}$ between points on them [12]:

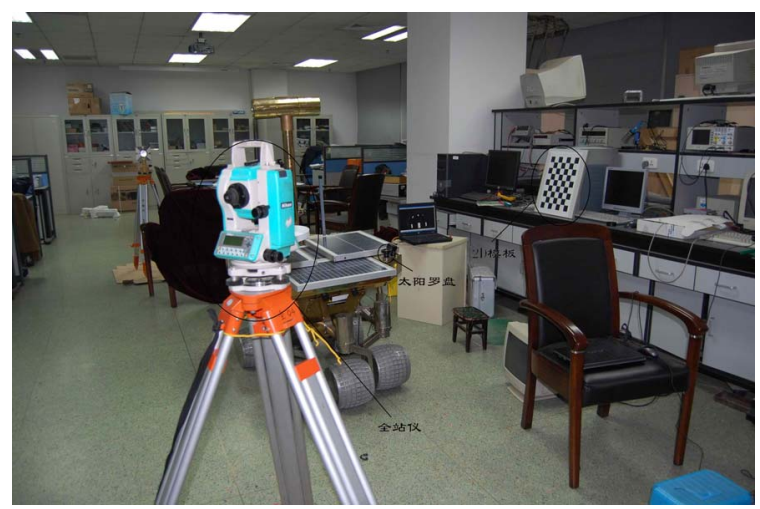

Figure 3. Configuration of the calibration.

$$
\lambda\left[\begin{array}{lll}
\sin \theta \cos \varphi & \sin \theta \sin \varphi & \cos \theta
\end{array}\right]^{\prime}=H_{p 2 f}\left[\begin{array}{lll}
X & Y & 1
\end{array}\right]^{\prime}
$$

where $\lambda$ is a scale factor, $\left[\begin{array}{lll}X & Y & 1\end{array}\right]^{\prime}$ is the homogenous coordinates of a control point on the calibration plane. For a control point and its image's back-projection, we also have

$$
\begin{aligned}
& s\left[\begin{array}{llll}
\sin \theta \cos \varphi & \sin \theta \sin \varphi & \cos \theta
\end{array}\right]^{\prime} \\
& =\left[\begin{array}{llll}
R & T
\end{array}\right]\left[\begin{array}{llll}
X & Y & 0 & 1
\end{array}\right]^{\prime} \\
& =\left[\begin{array}{lll}
r_{1} & r_{2} & T
\end{array}\right]\left[\begin{array}{lll}
X & Y & 1
\end{array}\right]^{\prime}
\end{aligned}
$$

where $r_{i}$ is the ith column of the rotation matrix $R, T$ is the translation vector, $s$ is a scale factor. Then we have $H_{p 2 f}=\left[\begin{array}{lll}r_{1} & r_{2} & T\end{array}\right]$ up to a scale. Furthermore,

$$
r_{1}=\lambda h_{1}, \quad r_{2}=\lambda h_{2}, \quad r_{3}=r_{1} \times r_{2}, T=\lambda h_{3}
$$

where $h_{i}$ is the ith column of the matrix $H_{p 2 f}$.

So the rotation $R_{p 2 f}$ and transformation $T_{p 2 f}$ between the fisheye camera coordinate system and the world coordinate system can be determined.

Similarly we can compute the homography $H_{p 2 t}$ from the calibration plane to the unit sphere of total station coordinate system, where the unit back-projection of the total station's measurement are distribute on. With decomposition similar to equation (11), the rotation $R_{p 2 t}$ and transformation $T_{p 2 t}$ between the total station coordinate system and the world coordinate system can also be determined.

The IMU can provide the Euler angle between the IMU coordinate system and the ground-plane coordinate system when IMU is still. With these measurements we can obtain the rotation $R_{I 2 g}$ between the IMU coordinate system and the ground-plane coordinate system. 
Considering that the total station coordinate system has been adjusted to be parallel to the ground-plane coordinate system, $R_{I 2 g}$ also represents the rotation between the IMU coordinate system and the total station coordinate system. So with the calibration plane in only one position, the rotation $R_{I 2 f}$ between the IMU coordinate system and the fisheye camera coordinate system can be obtained:

$$
R_{I 2 f}=R_{p 2 f} R_{p 2 t}^{-1} R_{I 2 g}
$$

For the fisheye camera and total station, there is a homography induced by the calibration plane between them. So we can directly compute the homography $H_{f 2 t}$ between the fisheye camera and total station. And compute the rotation $R_{f 2 t}$ and translation $T_{f 2 t}$ between them. Additionally we can use bundle adjustment [12] to obtain optimized estimation of $R_{f 2 t}$ by minimizing the squared distances between control points' unit back-projections

$$
\begin{aligned}
& \sum_{i}^{m} \sum_{j=1}^{n} d(m, \hat{m})^{2} \\
& \left(\begin{array}{lll}
\text { where } m=\left[\begin{array}{lll}
\sin \theta \cos \varphi & \sin \theta \sin \varphi & \cos \theta
\end{array}\right]^{\prime}, \\
\left.\hat{m}=\left[\begin{array}{lll}
\sin \hat{\theta} \cos \hat{\varphi} & \sin \hat{\theta} \sin \hat{\varphi} & \cos \hat{\theta}
\end{array}\right]^{\prime}\right)
\end{array}\right.
\end{aligned}
$$

using the Levenberg-Marquardt algorithm [13].

Then the rotation $R_{I 2 f}$ between the IMU coordinate system and the fisheye camera coordinate system can be computed with

$$
R_{I 2 f}=R_{f 2 t}^{-1} R_{I 2 g}
$$

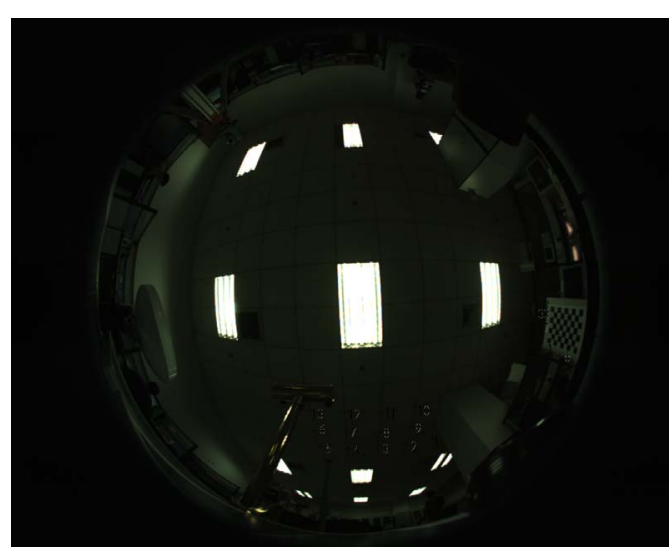

Figure 4. One image used to perform extrinsic calibration

\section{Experiments}

The proposed extrinsic calibration method has been tested on our lunar rover prototype shown in Figure 1. A NIKON DTM-300 total station is used. The fisheye camera is composed of a FUJINON FE185C046HA-1 fisheye lens and a Feith CANCAM camera. The view angle of the fisheye camera is 185 degrees. The intrinsic parameters of the fisheye camera shown in equation $(6,7,8)$ can be calibrated with a 2D planar pattern [9], [14]. The IMU used in our lunar rover prototype is a CROSSBOW IMU700CB IMU. Here we present the extrinsic calibration result with one example. The configuration of experiment is shown in Figure 3.

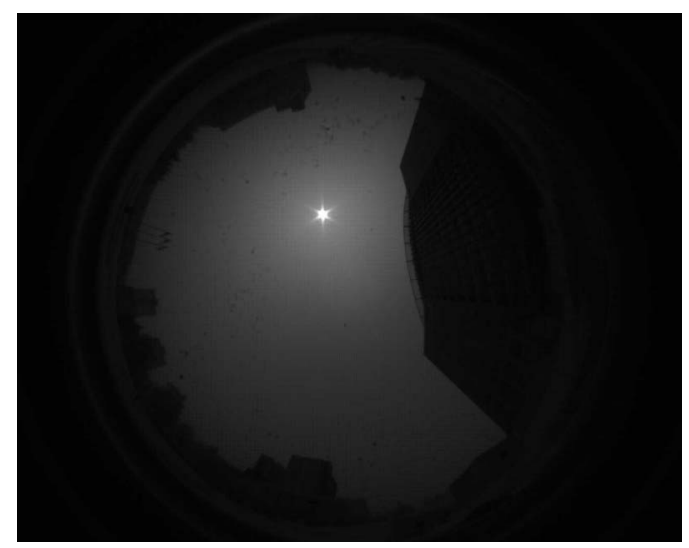

Figure 5. Image of Sun captured by the fisheye camera to validate the proposed method.

The used calibration pattern is a $6 \times 8$ checker board, and the size of a checker square is $600 \mathrm{~mm} \times 600 \mathrm{~mm}$. One image captured by the fisheye camera is shown in Figure 4. Extract control points in the image. Then compute the correspondent unit back-projections. Measure control points with the total station. And compute the homography $H_{f 2 t}$ induced by the calibration plane between the fisheye camera and the total station. Decompose the homography $H_{f 2 t}$ with equation (11), the rotation from the fisheye camera to the total station can be obtained:

$$
R_{f 2 t}=\left[\begin{array}{ccc}
0.9999 & 0.9973 & 0.0009 \\
0.0139 & 0.9999 & -0.0022 \\
-0.0008 & 0.0023 & 1.0000
\end{array}\right] .
$$

With the measurement of IMU, we can determine the rotation matrix from the ground-plane coordinate system to the IMU coordinate system

$$
R_{I 2 g}=\left[\begin{array}{ccc}
0.9997 & -0.0013 & -0.0243 \\
0 & 0.9985 & -0.0544 \\
0.0243 & 0.0544 & 0.9982
\end{array}\right] \text {. }
$$

Then the rotation from IMU coordinate system to the fisheye camera coordinate system is 


$$
R_{I 2 f}=\left[\begin{array}{ccc}
0.0378 & 0.9989 & 0.0272 \\
0.9916 & -0.0341 & -0.1247 \\
-0.1235 & 0.0318 & -0.9918
\end{array}\right]
$$

Table 1. Orientation results (in degrees) with calibrated parameters

\begin{tabular}{|c|c|c|c|}
\hline Elevation angle of & Azimuth angle & \multicolumn{2}{|c|}{ Orientation of lunar rover } \\
\cline { 3 - 4 } Sun $\theta$ & of Sun $\varphi$ & estimated & real \\
\hline 36.30 & -88.00 & 2.14 & 0.0 \\
\hline 60.79 & -125.13 & -188.06 & -185.0 \\
\hline 59.91 & -115.66 & -184.40 & -185.0 \\
\hline
\end{tabular}

To validate the extrinsic calibration method, we estimate the orientation of the lunar rover using celestial navigation method [15], [16] with the estimated extrinsic calibration parameters, the measurement of the fisheye camera and IMU. Use the fisheye camera capture images of Sun (one of them shown in Figure 5). Compute the azimuth angle $\varphi$ and the elevation angle $\theta$ of Sun in the fisheye camera coordinate system with equation $(6,7,8)$. Then with the estimated extrinsic calibration parameters, the orientation of Sun in the IMU (which is attached with lunar rover body) coordinate system can be computed. With the celestial navigation method [16], the orientation of the lunar rover in the absolute coordinate system can be computed. We also use compass to measure the orientation of the lunar rover and take it as the ground truth. Some results are shown in Table 1. We can see that there is little difference between the measured values and the ground truth. The error is less than 3.5 degrees. Errors may come from several sources. One is the image noise and the inaccuracy of Sun detection. Another is current rudimentary experimental setup: the ground truth is made with a compass with barely eye inspection. Even though, the experiments show the validity of the proposed extrinsic calibration method.

\section{Conclusions}

An extrinsic calibration method for a fisheye camera and an IMU which are in a general configuration where they can not observe each other is presented in this paper. The proposed method introduces a total station as a reference to perform calibration. The rotation between the fisheye camera and the total station is deduced via a planar pattern which is visible for both the camera and the total station. The total station can be adjusted to be parallel to the ground-plane coordinate system. The rotation between IMU coordinate system and the ground-plane coordinate system can be measured with IMU. Then the rotation between the fisheye camera and the IMU can be obtained. Orientation experiments with the proposed method on our lunar rover prototype show the validity of the proposed method. With this information the lunar rover prototype can robustly perform navigation task in complex environment by fusing measurements of the visual and inertial sensors.

\section{Acknowledgements}

This work is partially supported by the Open Project Program of the National Laboratory of Pattern Recognition (No.09-3-2) and the Scientific Research Foundation for the Young Scholars, Beijing University of Technology (No. 52002999200702).

\section{References}

[1] Grewal M.S, Weill L. R., and Andrews A. P., "Global positioning systems, intial navigation, and intergration", Wiley Inter-science, 2001.

[2] Shuster M.D., Dellinger W., "Spacecraft Attitude Determination and Control", in V.L.Pisacane (ed.), Fundamentals of Space Systems, 2nd ed., Oxford University Press, 236-325, 2005.

[3] Theophilos P., Konstantinos I. D., and et al., "Robust Estimation of Rigid-Body 3-D Motion Parameters Based on Point Correspondences", IEEE trans. on pattern analysis and machine intelligence, 10(4):541-549, 2000.

[4] Feng B., Bruyant P. P., Pretorius P. H., and et al., "Estimation of the Rigid-Body Motion from Three-Dimensional Images Using a Generalized Center-of-Mass Points Approach", IEEE trans. on Nucl Sci. 53(5): 2712-2718, 2006.

[5] Jekeli C., "Precision free-inertial navigation with gravity compensation by an onboard gradiometer", J. Guidance, Control and Dynamics, 29(3):704-713, 2006.

[6] David V., Alain M. and Nicolas P., "Using distributed magnetometers to increase IMU-based velocity estimation into perturbed area", In Proc. of $46^{\text {th }}$ IEEE Conference on Decision and Control, New Orleans, USA, 2007:4924-4931.

[7] Yang Y. and Farrell J. A., "Magnetometer and differential carrier phase GPS-aided INS for advanced vehicle control", IEEE Trans. Robotics Automation, 19(2):269-282, 2003.

[8] Goshen, L., Shimshoni, I., Anandan, P., and Keren, D., "Motion Recovery by Integrating over the Joint Image Manifold", International Journal of Computer Vision, 65(3):131-145, 2005. 
Proceedings of the Eighth International Conference on Machine Learning and Cybernetics, Baoding, 12-15 July 2009

[9] Kannala J., Brandt S. S., “A generic camera model and calibration method for conventional wide-angle, and fisheye lenses," IEEE trans. on pattern analysis and machine intelligence, 28(8):1335-1340, 2006.

[10] Tao T., Hu H. and Zhou H., "Integration of Vision and Inertial Sensors for 3D Arm Motion Tracking in Home-based Rehabilitation", International Journal of Robotics Research, 26(6): 607-624, 2007. KS, 2007.

[11] Zhou H., Hu H., Fei M.R., "Walking aids for elderly people: a systematic overview", Chinese Journal of Scientific Instrument, 29(1): 216-224, 2008.
[12] Hartley, R. I. and Zisserman, A., "Multiple View Geometry in Computer Vision", 2nd edition, Cambridge University Press, 2004.

[13] Kelley C. T., "Iterative Methods for Optimization", SIAM Frontiers in Applied Mathematics, no 18, 1999.

[14] Scaramuzza, D., Martinelli, A. and Siegwart, R., "A Toolbox for Easy Calibrating Omnidirectional Cameras", In: Proc. of IEEE/RSJ International Conference on Intelligent Robots and Systems, 2006.

[15] Susan Peterson Howell, "Practical Celestial Navigation", Mystic Seaport Museum, 1993.

[16] Favill J., "Prime of Celestial Navigation", READ BOOKS, 2007 